\title{
Ionic relaxation in polyethyleneimine-lithium bis(trifluoromethylsulfonyl) imide polymer electrolytes
}

\author{
İlknur Bayrak Pehlivan, ${ }^{1, a)}$ Roser Marsal, ${ }^{2}$ Peter Georén, ${ }^{2}$ Claes G. Granqvist, ${ }^{1}$ and \\ Gunnar A. Niklasson ${ }^{1}$ \\ ${ }^{1}$ Department of Engineering Sciences, The Angström Laboratory, Uppsala University, P.O. Box 534, \\ SE-75121 Uppsala, Sweden \\ ${ }^{2}$ ChromoGenics AB, Märstagatan 4, SE-75323 Uppsala, Sweden
}

(Received 26 April 2010; accepted 14 August 2010; published online 4 October 2010)

\begin{abstract}
Polymer electrolytes containing polyethyleneimine and different concentrations of lithium bis(trifluoromethylsulfonyl) imide were investigated by impedance spectroscopy at different temperatures. Two equivalent circuit models were compared for the bulk impedance response. The first one includes a conductive Havriliak-Negami (HN) element which represents ionic conductivity and ion pair relaxation in a single process, and the second model includes a dielectric HN element, which represents ion pair relaxation, in parallel with ion conductivity. Comparison of the two circuit models showed that the quality of the fit was similar and in some cases better for the conductive model. The experimental data follow the Barton-Nakajima-Namikawa relation, which relates the ion conductivity and the parameters of the relaxation. This indicates that ion conductivity and ion pair relaxation are two parts of the same process and should be described by the conductive model.
\end{abstract}

(C) 2010 American Institute of Physics. [doi:10.1063/1.3490133]

\section{INTRODUCTION}

Polymer electrolytes of high ionic conductivity, consisting of polymer and salt, have been extensively studied. ${ }^{1-3}$ They are of interest for providing ionic conduction in electrochemical devices such as electrochromic devices, ${ }^{4}$ batteries, ${ }^{5}$ fuel cells, ${ }^{6}$ and supercapacitors. ${ }^{7}$ In these applications the metallic cation is the electroactive species but in many cases the anion may give the major contribution to the conductivity. ${ }^{8}$ Ions in a polymer solute may be present either as free ions, contact ion pair dipoles, or alternatively diffusion ion pairs (sometimes called virtual dipoles). ${ }^{9}$ Understanding the contribution of these species, as well as that of larger ion aggregates, to the ionic conductivity is a major issue for obtaining an improved understanding of the electrical properties of polymer electrolytes. ${ }^{10}$

Dielectric spectroscopy is a very powerful technique for the investigation of the response to electric fields of various materials. A large number of models for the dielectric response have been advanced ${ }^{11-14}$ but there are still many unsolved and/or controversial questions. In recent years a renewed interest has been directed to ionic conduction processes in inorganic solids, ${ }^{15-17}$ and we feel that polymer electrolytes should also be discussed using similar concepts. Dielectric spectroscopy gives information about the macroscopic electrical properties of a material, which can be related to molecular quantities. ${ }^{18}$ The technique can conveniently be used to obtain the ionic conductivity but in this paper we study also a dielectric loss peak occurring in polymer electrolytes close to the onset of dc conductivity. This peak occurs only in salt-containing polymers and has previously been assigned to ion pair dipoles. ${ }^{10,19}$ The dielectric response of the polymer electrolyte can be modeled by an

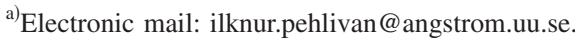

equivalent circuit consisting of electrical circuit elements such as capacitors and resistors or distributed circuit elements such as the Havriliak-Negami (HN) function. ${ }^{18,20}$ The equivalent circuit representation of a dielectric response is never unique, and one needs to have both physical knowledge and complementary information in order to find an appropriate model to represent the system.

Materials exhibiting both conductivity and dielectric relaxation can be modeled by dielectric or conductive models for the response. ${ }^{21} \mathrm{~A}$ general comparison of these types of response- that cannot usually be distinguished by data fitting alone-has been done by Macdonald. ${ }^{21-25}$ In a dielectric model the conductivity and the relaxation are viewed as different processes, and this would be consistent with the primary species being free ions and contact ion pairs. In a conductive model, the conductivity and relaxation are coupled and taken to arise from the same charge carriers; this situation would be consistent with the presence of diffusion ion pairs in a polymer electrolyte. Recent preliminary work ${ }^{26}$ has indicated that a conductive model is more appropriate for electrolytes with Li salt and polyethylene (PEO) or polypropylene (PPO) oxide. However, this previous analysis has some uncertainties because of the proximity of the ionic relaxation to the segmental relaxations of the polymer chains in these systems.

In this paper, we study the applicability of the dielectric and the conductive models to the impedance response of electrolytes containing polyethyleneimine (PEI) and lithium bis(trifluoromethylsulfonyl) imide (LiTFSI). PEI-LiTFSI was chosen because the ionic and polymeric relaxations are well separated, probably because PEI-LiTFSI has a lower conductivity than PEO-LiTFSI. Hence the possibility to distinguish conductive and dielectric models is better than for PEO based electrolytes. A few studies on impedance analysis of PEI aqueous solutions ${ }^{27}$ and PEI electrolytes ${ }^{28-30}$ have 
TABLE I. Glass transition temperatures $\left(T_{g}\right)$ of PEI-LiTFSI electrolytes with different salt concentrations.

\begin{tabular}{ccc}
\hline \hline$[\mathrm{N}]:[\mathrm{Li}]$ & $\begin{array}{c}C_{\text {salt }} \\
(\mathrm{mol} / \mathrm{l})\end{array}$ & $\begin{array}{c}T_{g} \\
\left({ }^{\circ} \mathrm{C}\right)\end{array}$ \\
\hline $400: 1$ & 0.06 & -61.5 \\
$200: 1$ & 0.13 & -61.2 \\
$100: 1$ & 0.25 & -62.8 \\
$50: 1$ & 0.50 & -60.8 \\
$20: 1$ & 1.25 & -51.8 \\
$10: 1$ & 2.51 & -44.8 \\
\hline \hline
\end{tabular}

been done before but a detailed investigation of the ionic relaxation appears not to have been carried out. A PEI based electrolyte has also been applied in an electrochromic device. ${ }^{30}$ Linear PEI, $\left(-\mathrm{CH}_{2} \mathrm{CH}_{2} \mathrm{NH}-\right)_{n}$, is the nitrogen ana$\log$ of PEO. ${ }^{31}$ Numerous studies of ionic conductivity and dielectric relaxation in PEO-LiTFSI electrolytes have been carried out earlier, for example, in Refs. 26 and 32-36.

\section{EXPERIMENTAL}

Branched PEI with average molecular weight 10.000 and purity 99\% was obtained from Alfa-Aesar. The Li salt LiTFSI [ $\mathrm{LiN}\left(\mathrm{CF}_{3} \mathrm{SO}_{2}\right)_{2}, 99.95 \%$ ] and anhydrous methanol $\left(\mathrm{CH}_{3} \mathrm{OH}, 99.8 \%\right)$ were supplied from Sigma-Aldrich and Merck, respectively. PEI was dried before use at $65^{\circ} \mathrm{C}$ and $10^{-1}$ mbar for $48 \mathrm{~h}$. LiTFSI was used after drying at $150{ }^{\circ} \mathrm{C}$ and $10^{-1}$ mbar for $72 \mathrm{~h}$. Methanol was used as received. All materials were stored dry $\left(<1\right.$ ppm $\left.\mathrm{H}_{2} \mathrm{O}\right)$ in a glove box under argon atmosphere.

LiTFSI was dissolved in methanol, and PEI was then added to the solutions and further stirring took place. The methanol was removed at $65^{\circ} \mathrm{C}$ and $10^{-1}$ mbar for $48 \mathrm{~h}$. PEI-LiTFSI electrolytes were prepared at molar ratios 400:1, 200:1, 100:1, 50:1, and 20:1. The relation between molar ratio and salt concentrations for the PEI-LiTFSI electrolytes is given in Table I.

Impedance measurements were performed between 20 and $60{ }^{\circ} \mathrm{C}$ on a Novocontrol BDC-N dielectric interface together with a Solartron 1260 frequency response analyzer with an applied ac voltage of $1 \mathrm{~V}$. Temperatures were controlled with an accuracy of $0.5{ }^{\circ} \mathrm{C}$. Temperature was kept constant during each measurement while frequency was swept over 48 points in the $10^{-2}-10^{7} \mathrm{~Hz}$ range. The measurement cell consisted of two parallel stainless steel electrodes with diameter of $20 \mathrm{~mm}$ separated by a ring-shaped Teflon spacer with $1 \mathrm{~mm}$ radial thickness and $3.1 \mathrm{~mm}$ height. All impedance measurements were done in the glove box under argon atmosphere. Real and imaginary parts of the permittivity were obtained from the measured capacitance by the equation

$$
\varepsilon^{*}(\omega)=\left[C^{*}(\omega)-C_{e}^{\prime}(\omega)\right] d / \varepsilon_{0} A,
$$

where $C^{*}(\omega)$ is the complex form of the measured capacitance of the sample, $C_{e}^{\prime}(\omega)$ is the real capacitance of the empty cell, $\omega$ is angular frequency, $d$ and $A$ are thickness and cross-sectional area of the sample, respectively, and $\varepsilon_{0}$ is the permittivity of vacuum.
Differential scanning calorimetry measurements on PEI and PEI-LiTFSI electrolytes were performed between -80 and $80{ }^{\circ} \mathrm{C}$. ${ }^{37}$ The electrolytes were found to have a glass transition between -45 and $-63{ }^{\circ} \mathrm{C}$ for different molar ratios. The glass transition temperature $\left(T_{g}\right)$ had a minimum and increased at high salt concentrations. Table I shows $T_{g}$ for different molar ratios.

\section{THEORY}

In the dielectric spectrum of polymers, one can identify bulk relaxation processes at high frequencies, which are related to the segmental motion of the polymer chains, and electrode polarization at low frequencies which is the result of the formation of electrical double layers at the polymer/ electrode interface. ${ }^{38}$ Information on the segmental relaxation of PEI has not been reported in the literature, as far as we know; it was not seen in our measurements and hence must be above $10 \mathrm{MHz}$. This high frequency relaxation can be approximately modeled by a series $R C$ circuit.

In the case of polymer electrolytes formed by dissolution of salts in polymers, adding ionic salts gives rise to an enhanced ionic conductivity and electrode polarization as well as to the appearance of an ion pair relaxation. ${ }^{39}$ Ion pairs are formed when ions of opposite electrical charge come together. It should be noted that dielectric relaxation spectroscopy is a powerful technique for investigation of ion-ion interactions. $^{40-43}$

In order to describe the ionic relaxation behavior quantitatively, one can use the very general empirical HN function. ${ }^{18}$ It can have the following two different forms: a dielectric HN (DHN, subscript $D$ ) function representing the ion pair relaxation, and a conductive $\mathrm{HN}(\mathrm{CHN}$, subscript $C)$ function representing the ionic conductivity and ion pair relaxation in a single process. The DHN function is given by

$$
C^{*}(\omega)=\frac{\Delta C}{\left[1+\left(i \omega \tau_{D}\right)^{U_{D}}\right]^{P_{D}}},
$$

where $\Delta C$ is difference between capacitance values at low and high frequencies, $\tau_{D}$ is the relaxation time, and $U_{D}$ and $P_{D}$ are power-law exponents. The $\mathrm{CHN}$ function is given by an expression analogous to Eq. (2) for the impedance, viz.,

$$
Z^{*}(\omega)=\frac{R_{C}}{\left[1+\left(i \omega \tau_{C}\right)^{U_{C}}\right]^{P_{C}}},
$$

where $R_{C}$ is the ionic resistance and the other symbols are introduced in analogy with those in Eq. (2). By using these types of the $\mathrm{HN}$ function, one can analyze the response of a system in the following two different ways: the first separates ionic conductivity and ion pair relaxation in a DHN response, and the second combines these two processes in a CHN response.

In our study, these two different types of the HN function were used in equivalent circuit models to fit the bulk response of the PEI-LiTFSI electrolytes. The DHN circuit in Fig. 1(a) consists of a series connection of a high-frequency resistance $\left(R_{D_{\infty}}\right)$ with a parallel connection of a highfrequency capacitance $\left(C_{D \infty}\right)$, a DHN element, and an ionic resistance $\left(R_{D}\right)$. The CHN circuit in Fig. 1(b) consists of a 
(a)
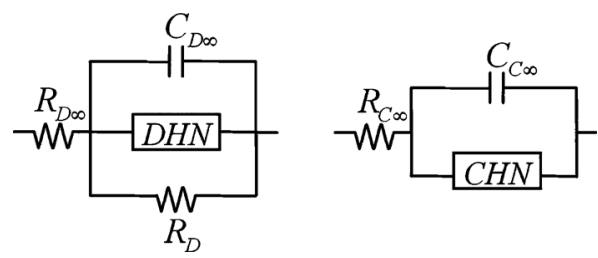

FIG. 1. Equivalent circuit models used for fitting data of PEI-LiTFSI electrolytes containing (a) DHN and (b) $\mathrm{CHN}$ circuit elements.

serial connection of a high-frequency resistance $\left(R_{C_{\infty}}\right)$ with a parallel connection of a high-frequency capacitance $\left(C_{C_{\infty}}\right)$, and a CHN element. The high-frequency resistance and capacitance approximately take into account the possible influence of polymer relaxations at frequencies exceeding 10 $\mathrm{MHz}$.

There is also a useful empirical relation, called the Barton-Nakajima-Namikawa (BNN) relation, ${ }^{44-46}$ between ionic conductivity $\sigma_{\text {ion }}$, dielectric relaxation, relaxation strength, and angular frequency of relaxation. It is given by

$$
\sigma_{\text {ion }}=F_{\mathrm{BNN}} \varepsilon_{0} \Delta \varepsilon \omega_{\max },
$$

where $F_{\mathrm{BNN}}$ is a numerical factor of the order of unity for ionic conductors, $\Delta \varepsilon$ is the dielectric relaxation strength, and $\omega_{\max }=2 \pi f_{\max }$ with $f_{\max }$ being the relaxation peak frequency corresponding to the location of the maximum in the dielectric loss. The BNN relation has been found to be valid for ion conducting glasses, ion conducting solids, and disordered solids. ${ }^{47-49}$

In the case of symmetrical relaxation peaks, for which the exponent $P_{D}$ in the DHN function is equal to one, the maximum in the dielectric loss occurs at $\omega_{\max } \tau_{D}=1$. For nonsymmetrical cases, the condition $\omega_{\max } \tau_{D}=1$ is modified to ${ }^{50}$

$$
\omega_{\max } \tau_{C, D}=\left(\frac{\sin \left(\frac{U_{C, D} \pi}{2\left(1+P_{C, D}\right)}\right)}{\sin \left(\frac{U_{C, D} P_{C, D} \pi}{2\left(1+P_{C, D}\right)}\right)}\right)^{1 / U_{C, D}} .
$$

Here we have also indicated a further generalization in order to determine $\omega_{\max }$ of the CHN function. It should be noted

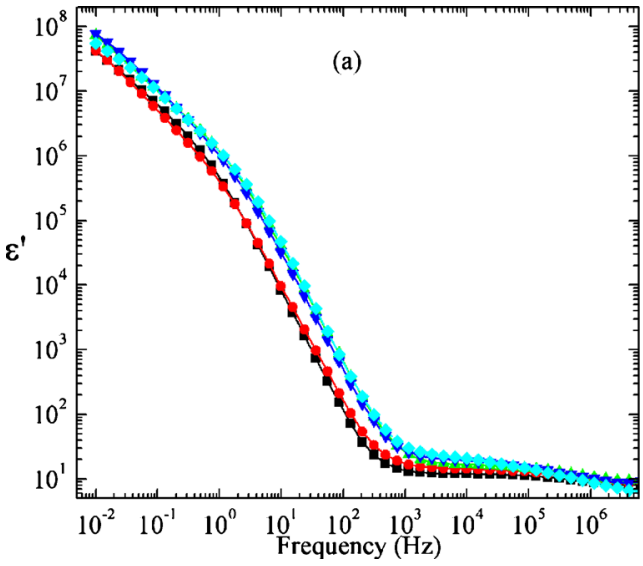

that $\omega_{\max }$ refers to the maximum of the dielectric loss for the DHN function and to the maximum of the imaginary impedance, or resistivity, for the CHN function.

The BNN relation shows that there is a connection between the ionic conductivity and the ion pair relaxation. This can be seen as a consequence of a scaling relation given by Dyre et al. ${ }^{49}$ and it also emerges directly from conductive models as shown by Macdonald. ${ }^{51}$ The implication is that the ionic conductivity and ion pair relaxation are due to the same conduction mechanism.

\section{RESULTS AND DISCUSSION}

The dielectric constant of PEI without salt was about 9, and its conductivity was approximately $10^{-7} \mathrm{~S} / \mathrm{cm}$ at a temperature of $20^{\circ} \mathrm{C}$. Formation of an electrolyte by adding $\mathrm{Li}$ salt to the PEI increased its dielectric constant and ionic conductivity up to 18 and $3 \times 10^{-6} \mathrm{~S} / \mathrm{cm}$, respectively, depending on the salt concentration.

Figure 2 shows the real and imaginary parts of the dielectric permittivity of the PEI-LiTFSI electrolytes at room temperature for different salt concentrations. Electrode polarization is seen in the real part of the permittivity below a frequency of $10^{3} \mathrm{~Hz}$, and bulk relaxation is apparent above this frequency. The bulk permittivity has similar values at different salt concentrations. When the salt concentration was enhanced, the strength of the bulk relaxation increased. Dielectric loss increased as $\omega^{-1}$, which is the characteristic behavior of ionic conductivity. The dc conductivity increased as a function of salt concentration, exhibited a maximum at intermediate concentration and then decreased. It dominated the dielectric loss spectra of all our PEI-LiTFSI polymer electrolytes, and therefore it was difficult to observe dielectric loss peaks by means of subtracting the dc conductivity contribution. However, they were inferred from the equivalent circuit fitting, as described below.

The time-temperature superposition principle implies that the temporal (or frequency) response of a material exhibits the same shape at different temperatures. Permittivity spectra of PEI-LiTFSI electrolytes at different temperatures were shifted along the axes in order to investigate whether they had the same shape. In Fig. 3 the curves at all of the

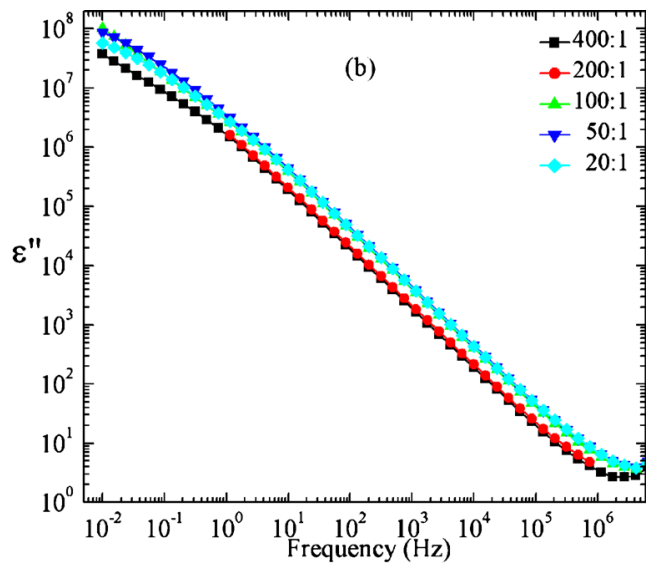

FIG. 2. (Color online) (a) Real $\left(\varepsilon^{\prime}\right)$ and (b) imaginary $\left(\varepsilon^{\prime \prime}\right)$ permittivity as a function of frequency for PEI-LiTFSI electrolytes with different salt concentrations at $20{ }^{\circ} \mathrm{C}$. 


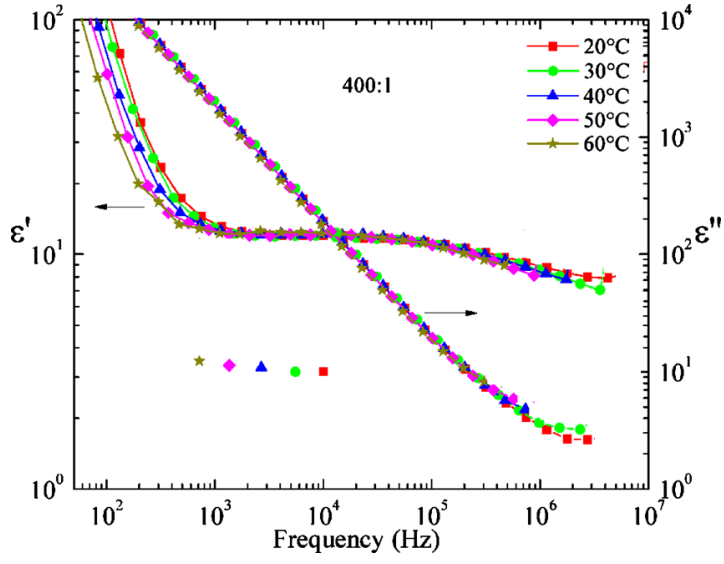

FIG. 3. (Color online) Time-temperature superposition of real and imaginary dielectric permittivity for PEI-LiTFSI electrolytes at 400:1 molar ratio. The symbols shown below the graphs indicate the same real and imaginary dielectric permittivity at a frequency of $10^{4} \mathrm{~Hz}$ for each of the superposed graphs.

different temperatures were shifted onto the room temperature curve. It is seen that all data fell on a single curve for the bulk response, while the electrode polarization displays a different behavior. This shows that the bulk permittivity data taken at different temperatures are related to one another by the time-temperature superposition principle. The bulk relaxation part shifts to higher frequencies with increasing temperature. Because of this, the bulk properties were seen in a much narrower frequency range at high temperatures than at low temperatures. There were fewer data points in the former case and, as a consequence, fits at room temperature gave more accurate parameter values.

To investigate the bulk response, impedance spectra of PEI-LiTFSI electrolytes at different salt concentration were fitted, in the frequency range above $1 \mathrm{kHz}$, to the equivalent circuit models introduced above. The fitting used the leastsquares method, which is useful when comparing the goodness of fit of different models to a single data set, since it is proportional to the average percentage error between the original data points and the calculated values of the fitting. The least-squares fittings did not differ significantly for the two models; specific values were less than 0.02 for both, but somewhat lower for the $\mathrm{CHN}$ circuit at $20{ }^{\circ} \mathrm{C}$. Therefore one can state that the quality of the fit was similar, or even slightly better, for the CHN circuit despite the fact that it has one parameter less.

The measured data for the PEI-LiTFSI electrolytes with different salt concentration and at room temperature are shown together with the CHN circuit model fits in Fig. 4 in the form of a Nyquist plot. Fits to the DHN circuit were virtually identical. The semicircles decreased with increasing salt concentration until, at a molar ratio of 50:1, the righthand end of the semicircle intercepted the $Z^{\prime}$ axis at the smallest value. The ionic resistance is lowest at that concentration. The maximum of $Z^{\prime \prime}$ decreased for increasing salt concentration.

In order to further justify the use of a conductive model to describe the PEI-LiTFSI electrolytes, the relation between the ionic conductivity, relaxation strength, and relaxation fre-

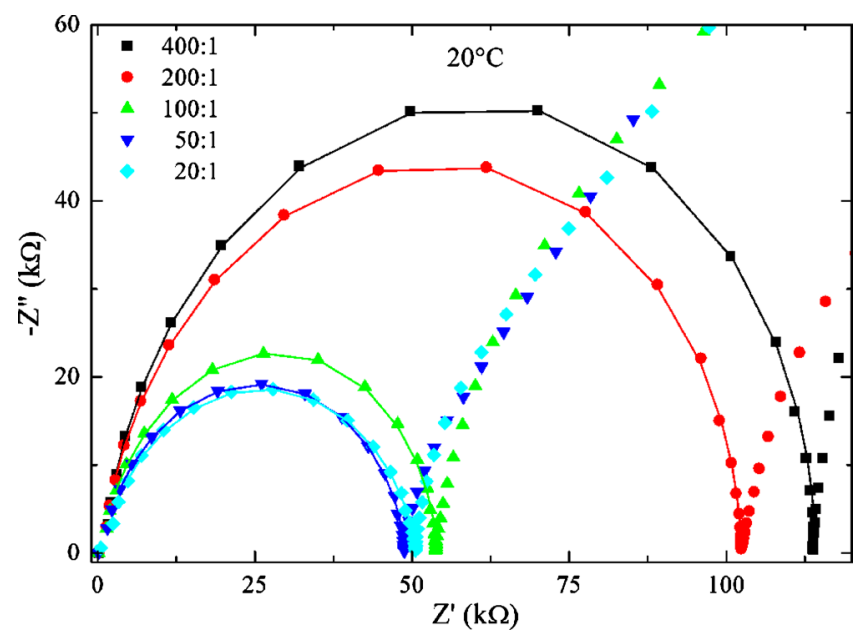

FIG. 4. (Color online) Imaginary impedance vs real impedance at $20^{\circ} \mathrm{C}$ for PEI-LiTFSI electrolytes with different salt concentrations. Experimental impedance results are shown as symbols and impedance fits obtained by using the $\mathrm{CHN}$ model as lines.

quency was investigated by means of the BNN relation in Eq. (4). The $F_{\mathrm{BNN}}$ factor was derived by using the modified $\omega_{\max }$ as obtained numerically via Eq. (5) from parameters for the DHN function. The salt concentration dependence of $F_{\mathrm{BNN}}$ for the PEI-LiTFSI electrolytes is shown in Fig. 5 for $20{ }^{\circ} \mathrm{C}$. The values of $F_{\mathrm{BNN}}$ lay approximately between 1 and 2.5. As a consequence of the time-temperature superposition relation, similar behaviors of $F_{\mathrm{BNN}}$ were obtained at the other temperatures. It should be noted that values of $F_{\mathrm{BNN}}$ have recently been computed by Macdonald for some conductive response models. ${ }^{52}$ Our values are close to the theoretical ones for a stretched exponential (Kohlrausch) function, which hence may be an interesting alternative to the HN element in our case.

Figure 6 shows the parameters of the CHN element versus salt concentration at different temperatures. The ionic resistance, shown in Fig. 6(a), displays a slight decrease with increasing salt concentration and is significantly reduced at increased temperatures. The relaxation time is almost constant as a function of salt concentration but has a minimum at medium concentration as seen from Fig. 6(b). It also decreases with increasing temperature. One of the power-law

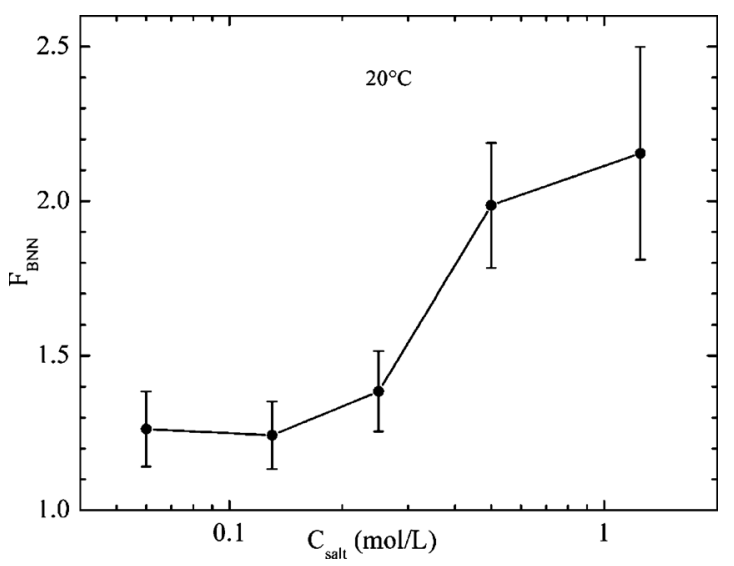

FIG. 5. BNN-factor $F_{\mathrm{BNN}}$ as a function of salt concentration for PEI-LiTFSI electrolytes at $20^{\circ} \mathrm{C}$. 

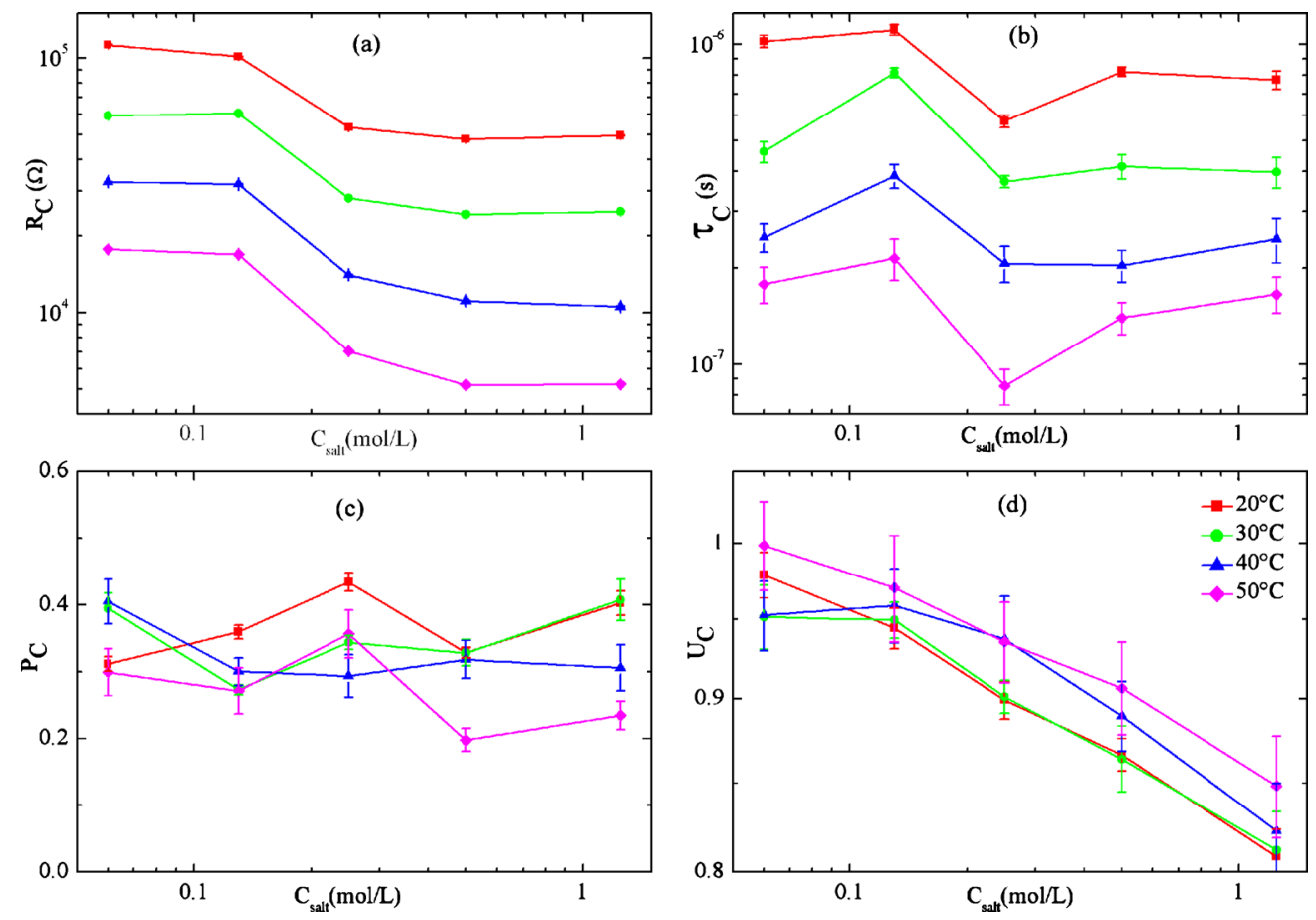

FIG. 6. (Color online) Salt concentration dependence of (a) ionic resistance, (b) relaxation time, (c) power-law exponent $P_{C}$, and (d) power-law exponent $U_{C}$ of the CHN function for PEI-LiTFSI electrolytes at different temperatures.

exponents, $P_{C}$, does not show a general trend with salt concentration, as apparent from Fig. 6(c), but the other exponent, $U_{C}$, decreases with increasing concentration; cf. Fig. 6(d). The values of $P_{C}$ are lower than 0.5 . The parameter $U_{C}$ is close to unity at low salt concentrations and decreases with increasing salt concentration.

The activation energies of ionic conductivity $E_{i}$ and of ion pair relaxation $E_{r}$ were obtained by fitting an Arrhenius equation to the ionic conductivity and the modified $\omega_{\max }$, which was obtained from Eq. (5) using the parameters of the CHN function, respectively. These energies are found to be of the same order of magnitude as apparent from Fig. 7. We also know from the time-temperature superposition relation that the shape of the ac response remained invariant under

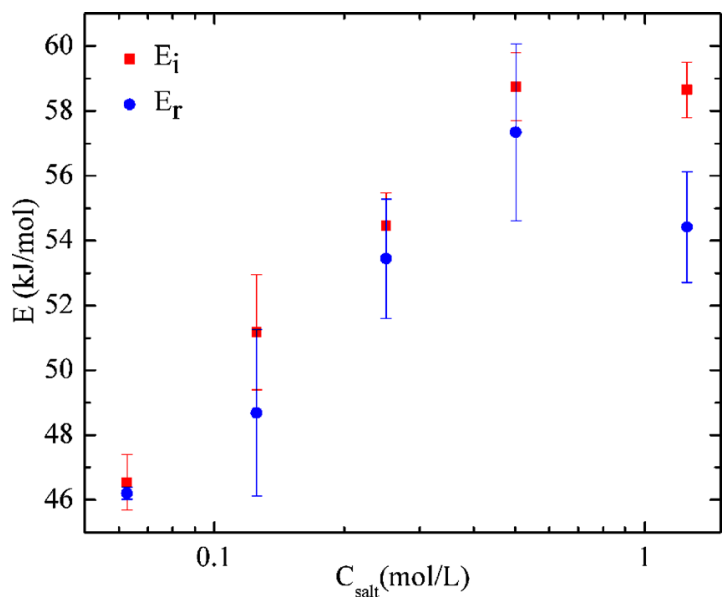

FIG. 7. (Color online) Salt concentration dependence of activation energy obtained by means of temperature dependence of ionic conductivity and relaxation time for PEI-LiTFSI electrolytes; they are shown as $E_{i}$ and $E_{r}$, respectively. temperature. Thus one can state that ionic conductivity and relaxation frequency have nearly the same activation energy. This shows that they should be combined to a single process, as is the case in the CHN function.

\section{CONCLUSION}

This paper examined the role of ion-pair relaxation and ionic conductivity in the ion transport process for PEILiTFSI electrolytes at different salt concentrations and temperatures. We compared $\mathrm{CHN}$ and DHN circuit models for these electrolytes. The conductive model, that includes ionpair relaxation and ionic conductivity in a single process, gave similar and in some cases better fits to experimental data. Second, we obtained good agreement with the BNNrelation, which indicates a connection between ionic conductivity and ion-pair relaxation. Finally, we obtained activation energies of ionic conductivity and ion-pair relaxation, both having the same value. These results lead us to the conclusion that ionic conductivity and ion-pair relaxation should be viewed as two parts of the same conduction process.

\section{ACKNOWLEDGMENTS}

This work was partially supported by a grant from the Swedish Research Council for Environment, Agricultural Sciences, and Spatial Planning (FORMAS). One of the authors (I.B.P.) gratefully acknowledges Professor Fatma Tepehan for making the scientific collaboration behind this work possible. We are thankful to Professor J. Ross Macdonald for valuable discussions on the BNN relation and for providing data of $F_{\mathrm{BNN}}$ for a number of conductive models. 
${ }^{1}$ Polymer Electrolyte Reviews, edited by J. R. MacCallum and C. A. Vincent (Elsevier Applied Science, London, 1987), Vol. 1.

${ }^{2}$ M. Armand, W. Gorecki, and R. Andreani, in Proceedings of the Second International Symposium on Polymer Electrolytes, edited by B. Scrosati (Elsevier Applied Science, London, 1990), p. 91.

${ }^{3}$ P. G. Bruce and C. A. Vincent, J. Chem. Soc., Faraday Trans. 89, 3187 (1993).

${ }^{4}$ G. A. Niklasson and C. G. Granqvist, J. Mater. Chem. 17, 127 (2007).

${ }^{5}$ M. B. Armand, Annu. Rev. Mater. Sci. 16, 245 (1986).

${ }^{6}$ Polymer Electrolyte Fuel Cell Durability, edited by F. N. Büchi, M. Inaba, and T. J. Schmidt (Springer, New York, 2009).

${ }^{7}$ P. Staiti and F. Lufrano, Electrochim. Acta 55, 7436 (2010).

${ }^{8}$ M. M. Doeff, P. Georén, J. Qiao, J. Kerr, and L. C. De Jonghe, J. Electrochem. Soc. 146, 2024 (1999).

${ }^{9}$ R. M. Fuoss, J. Solution Chem. 7, 771 (1978).

${ }^{10}$ I. Albinsson, B. E. Mellander, and J. R. Stevens, Solid State Ionics 72, 177 (1994).

${ }^{11}$ A. K. Jonscher, Dielectric Relaxation in Solids (Chelsea Dielectrics, London, 1983)

${ }^{12}$ G. A. Niklasson, J. Appl. Phys. 62, R1 (1987).

${ }^{13}$ J. R. Macdonald, J. Appl. Phys. 62, R51 (1987)

${ }^{14}$ J. R. Macdonald and J. C. Phillips, J. Chem. Phys. 122, 074510 (2005).

${ }^{15}$ J. R. Macdonald, J. Appl. Phys. 90, 153 (2001).

${ }^{16}$ J. C. Dyre, P. Maass, B. Roling, and D. L. Sidebottom, Rep. Prog. Phys. 72, 046501 (2009).

${ }^{17}$ D. L. Sidebottom, Rev. Mod. Phys. 81, 999 (2009).

${ }^{18}$ S. Havriliak, Jr. and S. J. Havriliak, Dielectric and Mechanical Relaxation in Materials: Analysis, Interpretation, and Application to Polymers (Hanser, Munich, 1997).

${ }^{19}$ L. R. A. K. Bandara, M. A. K. L. Dissanayake, M. Furlani, and B.-E Mellander, Ionics 6, 239 (2000).

${ }^{20}$ J. R. Macdonald and W. B. Johnson, in Impedance Spectroscopy: Theory, Experiment, and Applications, edited by E. Barsoukov and J. R. Macdonald (Wiley-Interscience, Hoboken, 2005), p. 13.

${ }^{21}$ J. R. Macdonald and M. K. Brachman, Rev. Mod. Phys. 28, 393 (1956).

${ }^{22}$ J. R. Macdonald, J. Non-Cryst. Solids 197, 83 (1996).

${ }^{23}$ J. R. Macdonald, J. Non-Cryst. Solids 210, 70 (1997).

${ }^{24}$ J. R. Macdonald, Braz. J. Phys. 29, 332 (1999).

${ }^{25}$ J. R. Macdonald, J. Chem. Phys. 118, 3258 (2003).

${ }^{26}$ M. Furlani, C. Stappen, B.-E. Mellander, and G. A. Niklasson, J. NonCryst. Solids 356, 710 (2010).

${ }^{27}$ U. Kaatze, O. Götman, R. Podbielski, and R. Pottel, J. Mol. Liq. 37, 127 (1988).

${ }^{28}$ C. S. Harris, M. A. Ratner, and D. F. Shriver, Macromolecules 20, 1778 (1987).
${ }^{29}$ B. Unal, R. J. Klein, K. R. Yocca, and R. C. Hedden, Polymer 48, 6077 (2007).

${ }^{30} \mathrm{H}$. Hu, B. E. Ortíz-Aguilar, and L. Hechavarría, Opt. Mater. (Amsterdam, Neth.) 29, 579 (2007).

${ }^{31}$ J. M. G. Cowie, in Polymer Electrolyte Reviews, edited by J. R. MacCallum and C. A. Vincent (Elsevier Applied Science, London, 1987), Vol. 1, p. 69.

${ }^{32}$ P. A. R. D. Jayathilaka, M. A. K. L. Dissanayake, I. Albinsson, and B.-E. Mellander, Electrochim. Acta 47, 3257 (2002).

${ }^{33}$ J. R. Dygas, B. Misztal-Faraj, Z. Florjanczyk, F. Krok, M. Marzantowicz, and E. Zygadlo-Monikowska, Solid State Ionics 157, 249 (2003).

${ }^{34}$ M. Marzantowicz, J. R. Dygas, F. Krok, Z. Florjanczyk, and E. ZygadłoMonikowska, J. Non-Cryst. Solids 352, 5216 (2006).

${ }^{35}$ M. Marzantowicz, J. R. Dygas, F. Krok, Z. Florjanczyk, and E. ZygadłoMonikowska, J. Non-Cryst. Solids 353, 4467 (2007).

${ }^{36}$ J. R. Dygas, Solid State Ionics 176, 2065 (2005).

${ }^{37}$ İ. B. Pehlivan, R. Marsal, G. A. Niklasson, C. G. Granqvist, and P. Georén, "PEI-LiTFSI electrolytes for electrochromic devices: Characterization by differential scanning calorimetry and viscosity measurements," Sol. Energy Mater. Sol. Cells (to be published).

${ }^{38}$ A. K. Jonscher, Dielectric Relaxation in Solids (Chelsea Dielectrics London, 1983), p. 174

${ }^{39}$ C. A. Vincent, Prog. Solid State Chem. 17, 145 (1987).

${ }^{40}$ J. Barthel, R. Buchner, P.-N. Eberspächer, M. Münsterer, J. Stauber, and B. Wurm, J. Mol. Liq. 78, 83 (1998).

${ }^{41} \mathrm{R}$. Buchner, in Novel Approaches to the Structure and Dynamics of Liquids: Experiments, Theories and Simulations, edited by J. Samios and V. A. Durov (Kluwer, Dordrecht, 2002), p. 265.

${ }^{42}$ R. Buchner, T. Chen, and G. Hefter, J. Phys. Chem. B 108, 2365 (2004).

${ }^{43}$ B. Wurm, C. Baar, R. Buchner, and J. Barthel, J. Mol. Liq. 127, 14 (2006).

${ }^{44}$ H. Namikawa, J. Non-Cryst. Solids 18, 173 (1975).

${ }^{45}$ T. Nakajima, 1971 Annual Report of Conference on Electrical Insulation and Dielectric Phenomena (National Academy of Sciences, Washington, D.C., 1972), p.168.

${ }^{46}$ J. L. Barton, Verres Refract. 20, 328 (1966); C. R. Acad. Sci. (Paris) 264, 1139 (1967).

${ }^{47}$ D. L. Sidebottom, Phys. Rev. Lett. 82, 3653 (1999).

${ }^{48}$ D. L. Sidebottom, B. Rolling, and K. Funke, Phys. Rev. B 63, 024301 (2000).

${ }^{49}$ J. C. Dyre and T. B. Schrøder, Rev. Mod. Phys. 72, 873 (2000).

${ }^{50}$ A. Boersma, J. van Turnhout, and M. Wübbenhorst, Macromolecules 31, 7453 (1998).

${ }^{51}$ J. R. Macdonald, Phys. Rev. B 71, 184307 (2005).

${ }^{52}$ J. R. Macdonald, J. Appl. Phys. 107, 101101 (2010). 